\title{
Effects of Interferential Current Treatment on Pain, Functional Ability, and Health-Related Quality of Life in Chronic Stroke Patients with Lumbago; A Randomized Controlled Study
}

\author{
Kyoung-Sim Jung, PT $\cdot$ Tae-Sung $\mathrm{In}, \mathrm{PT}^{\dagger}$ \\ Department of Physical Therapy, Gimcheon University
}

Received: November 18, 2019 / Revised: November 19, 2019 / Accepted: December 17, 2019

(c) 2020 J Korean Soc Phys Med

\section{| Abstract |}

PURPOSE: This study examined the efficacy of an interferential current (IFC) treatment on the improvement of pain, disability, and quality of life in stroke patients with lumbago.

METHODS: A double-blind, randomized clinical trial was conducted on 40 stroke patients with lumbago. The patients were allocated randomly into two groups: the IFC treatment group $(n=20)$ and the placebo treatment group $(n=20)$. The IFC group received 30 minutes of IFC treatment on the lumbar region, while the placebo group received IFC treatment but without real electrical stimulation. The intervention was administered five days a week for four weeks. The primary outcomes of pain intensity were measured using a visual analogue scale. The secondary measurements included the Barthel Index, Oswestry Disability Index (ODI), and

$\uparrow$ Corresponding Author : Tae-Sung In in8386@naver.com, https://orcid.org/0000-0002-7672-5150

This is an Open Access article distributed under the terms of the Creative Commons Attribution Non-Commercial License (http://creativecommons.org/licenses/by-nc/3.0) which permits unrestricted non-commercial use, distribution, and reproduction in any medium, provided the original work is properly cited. health-related quality of life (HRQoL).

RESULTS: The measurements were conducted before and after the two-week intervention period. Compared to the placebo treatment group, the IFC treatment group showed significantly greater improvement in the pain intensity $(p<.05)$, ODI $(p<.05)$, and SF-36 $(p<.05)$ at the end of the intervention. No significant differences in the Barthel Index were found between the two groups.

CONCLUSION: These findings show that an IFC treatment can improve pain, functional ability, and quality of life, highlighting the benefits of somatosensory stimulation from IFC in stroke patients with lumbago.

Key Words: Electric stimulation therapy, Pain, Activities of daily living, Quality of life, Stroke

\section{Introduction}

Stroke can cause severe and long-term functional disabilities in the elderly [1,2], of which pain is quite common (prevalence, 11\%-53\%) [3-6]. In addition to shoulder pain, back and lower extremity pain are also observed in stroke patients. In some cases, patients have more than one type of post-stroke pain. $[4,7,8]$. 
Regardless of the patient's age, disease severity, and functional ability, pain adversely affects the rehabilitation outcome in stroke patients [9]. Lumbago has been shown to reduce the health-related quality of life (HRQoL) and the balance and gait of affected individuals [10]. In a recent study, Köseoğlu et al. [11] reported that appropriate and early interventions for pain management are critical in minimizing the functional disability. Although patients with stroke may experience pain in various parts of the body, including the shoulder, wrist, back, and lower extremities, most studies on pain in these patients focused on the shoulder $[3,4,7,8]$. Electrotherapy, which is a non-invasive and non-pharmaceutical intervention for pain reduction, involves mainly the use of transcutaneous electrical nerve stimulation and interferential current (IFC) [12]. IFC utilizes a low frequency $(0-250 \mathrm{~Hz})$ current, which is generated from different cross-interfering mid-frequency (around $4000 \mathrm{~Hz}$ ) electrical currents [13]. This method can reduce the skin resistance, thereby enabling the current to penetrate deeper into the tissues without causing discomfort $[13,14]$. IFC not only stimulates the sensory nerve fibers to reduce pain but also relaxes the muscles and facilitates blood circulation [15,16]. This method can improve both pain and functionality significantly in patients with musculoskeletal conditions of the knee, shoulder, and back. Suriya-amarit et al. [17] reported significant improvements in stroke patients with shoulder pain and ROM after IFC use. On the other hand, to the best of the authors' knowledge, the effectiveness of this method in stroke patients with back pain has not been studied. This study examined the effects of IFC on pain, functional ability, and HRQoL in stroke patients with lumbago.

\section{Materials and Methods}

\section{Participants}

Forty stroke patients with LBP aged between 19 and 65 years were recruited for this study from J Hospital in
Gyeonggi-do. The subjects had experienced low back pain for three months or longer, had a visual analogue scale score of four or higher, could maintain their standing posture independently for 30 seconds or longer and could understand and follow the instructions given by the researcher (MMSE $>24$ points) [18]. The following patients were excluded: patients with radiculopathy; those with contraindications to electrotherapy, lumbar fracture, or surgery; those taking medications that can affect the posture control, gait, or pain; and those who received any physical therapy eight weeks before recruitment in this study [19]. Table 1 lists the common characteristics of the participants in this study. Informed consent was obtained voluntarily from all patients before participation in this study, which was approved by the Institutional Review Board of Gachon University (1044396-201801-HR-009-01). This study used G-power 3.1.7 to calculate the sample size, which was determined based on the ability to detect clinically significant improvement in the outcome measures from a pilot study (17 patients with IFC and 17 patients with placebo (FC), and the effect size and alpha error were set to 0.8 and 0.05 , respectively. According to the analysis, at least 20 subjects for each group were necessary to make an acceptable group size.

\section{Experimental procedure}

This study was designed as a double-blinded, randomized controlled trial (RCT). The participants were evaluated before and one day after training for four weeks by three well-trained physical therapists, who were blinded to the subjects and the purpose of this study. Forty subjects were assigned randomly to either the experimental group $(n=20)$ or the control group $(n=20)$ using a selection envelope. A person who was not involved in the study picked out a number (either 1 or 2 ) from a sealed envelope for unbiased randomization. The treatment lasted for 30 minutes a day, five times a week for four weeks. The sub jects in the IFC treatment group received electrical 
Table 1. General Characteristics of the Participants

\begin{tabular}{|c|c|c|c|}
\hline Variable & IFC group $(n=20)$ & Placebo group $(n=20)$ & p-valuea \\
\hline \multicolumn{4}{|l|}{ Sex } \\
\hline Male & 6 & 9 & $.327^{\mathrm{a}}$ \\
\hline Female & 14 & 11 & \\
\hline Age & $48.05 \pm 10.65^{\mathrm{a}}$ & $52.40 \pm 10.83$ & $.208^{\mathrm{b}}$ \\
\hline Weight $(\mathrm{kg})$ & $61.95 \pm 13.94$ & $63.90 \pm 12.86$ & $.648^{\mathrm{b}}$ \\
\hline Height $(\mathrm{cm})$ & $164.63 \pm 16.01$ & $167.10 \pm 14.73$ & $.528^{\mathrm{b}}$ \\
\hline Duration of Pain (month) & $14.95 \pm 7.04$ & $16.80 \pm 5.94$ & $.375^{\mathrm{b}}$ \\
\hline Duration of Dtroke(month) & $30.16 \pm 14.45$ & $36.27 \pm 16.91$ & $.272^{\mathrm{b}}$ \\
\hline MMSE & $25.95 \pm 7.043$ & $26.56 \pm 7.04$ & $.119^{\mathrm{b}}$ \\
\hline
\end{tabular}

MMSE, mini-mental status examination; VAS, visual analogue Scale; ODI, Oswestry disability index for low back pain Values are expressed as mean \pm standard deviation (SD).

${ }^{a}$ Chi-square test Independent t-test.

${ }^{b}$ Independent t-test.

stimulation for 30 minutes, while those in the placebo treatment group received non-electrically stimulated IFC for the same amount of time. All the subjects received conventional therapy for one hour a day, five times a week, for four weeks. Conventional therapy consisted of a range of motion exercises, mat exercises, and gait training for one hour per day. All subjects were evaluated for their pain, functional ability, and health-related quality of life after the four-week training period

\section{Intervention}

The subjects were asked to expose their waist while lying on their side, and they were electrically stimulated for 30 minutes using an IFC treatment device (IF-7P; ITO CO., Japan). Four electrodes were attached so that they crossed at the lumbar 1 and 5 height. The carrier frequency was set to $4000 \mathrm{~Hz}$, and the amplitude-modulated frequency was $80 \mathrm{~Hz}$. The current electricity intensity was adjusted to an individual tolerable level of pins-and-needles sensation at a level of invisible muscle contraction. Furthermore, the mediator was asked to observe whether motion due to muscle contraction had occurred in the

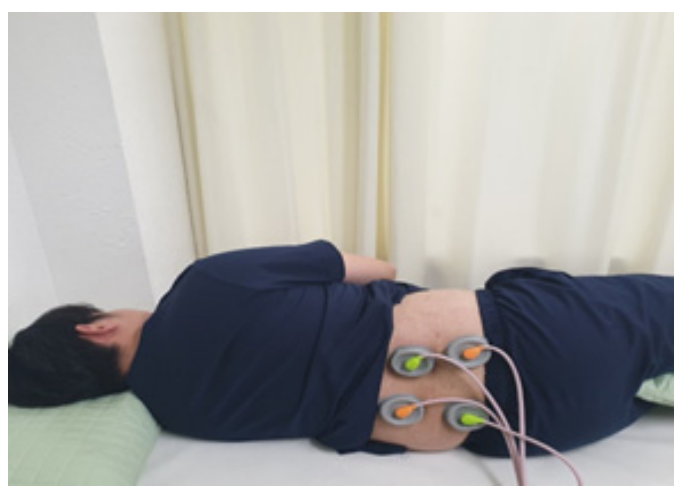

Figure 1. IFC treatment.

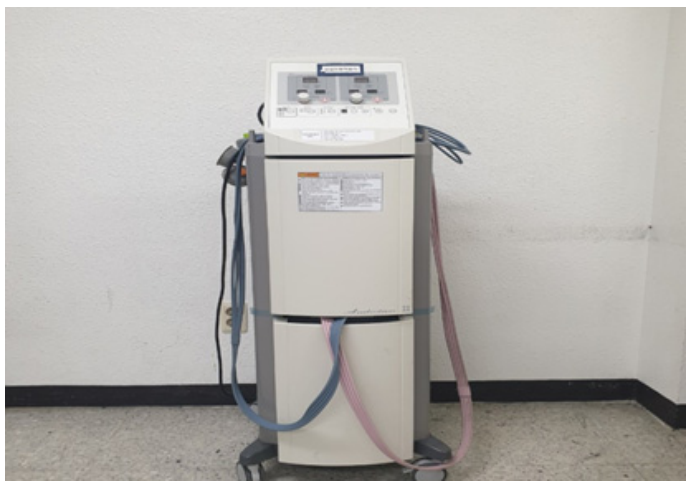

Figure 2. Treatment device. 
participant. In the placebo treatment group, the electrodes were attached at the same location, but electrical stimulation was not applied.

\section{Outcome measures}

\section{1) Pain intensity}

A visual analogue scale (VAS) was used to measure the pain intensity. The score for each item ranged from 0 to 10 . The VAS has high sensitivity and is proportional to the level of pain. The VAS is a commonly used assessment instrument for pain. This is the most widely used measurement method for pain with good reliability $[20,21]$. In this study, the pain at rest and during anterior trunk flexion in the standing position was measured. For the anterior trunk flexion, the same maximum distance before the intervention was applied after the intervention to measure the pain.

\section{2) Functional ability}

The subject's functional ability was measured using the Barthel Index and the Oswestry Disability Index (ODI). The Barthel Index is a measure of independence in the activities of daily living (ADL). This index consists of 10 items: feeding, bathing, personal hygiene (grooming), dressing, toileting, transferring, walking on a level surface, going up and down stairs, and continence of bowel and bladder [22]. The score ranged from 0 to 100 . A higher score represents a higher degree of independence in performing the ADL. The Barthel Index demonstrated good intra-rater reliability [23]. The Oswestry Disability Index (ODI) is one of the disability measures for low back pain. The ODI is a self-administered questionnaire consisting of 10 items, including pain intensity, sleep quality, ability to care for oneself, ability to walk, ability to sit, ability to stand, lifting, sexual function, social life, and ability to travel. The index provides a score out of 50, which in turn may be calculated as a percentage of disability value $(0 \%-100 \%)$ [24]. The test-retest reliability of the ODI is 0.92 , which is very high [25].

\section{3) Health-related quality of life}

The Short-Form 36 Questionnaire was used to evaluate the health-related quality of life [26]. The questionnaire contained 36 items, and the response to each item was converted to a score of 0 to 100 . The individual items are integrated into eight sub-domain scores (physical functioning, role limitations due to physical health, role limitations due to emotional problems, energy/fatigue, emotional well-being, social functioning, pain, and general health), and sub-domain scores are used to generate the physical and mental health scores. A higher score indicates a better health status.

\section{Data analysis}

The normality of the variables was assessed using the Shapiro-Wilk test. An independent t-test (for continuous variables) and a chi-square test (for categorical variables) were used to compare the baseline characteristics of the subjects in the IFC and placebo stimulation groups. A paired $\mathrm{t}$-test was used for the intra-group comparison, and an independent $\mathrm{t}$-test was used for the inter-group comparison. The level of statistical significance was set to 0.05 . SPSS 12.0 was used for statistical analysis.

\section{Results}

No subject dropped out after the training, and there were no serious problems, such as cutaneous inflammation, pain, and infection, following the sit-to-stand training with/without IFC application.

No significant differences in the general characteristics were found between the IFC and placebo stimulation groups before treatment (Table 1). The training caused a more significant decrease in the VAS for pain in the IFC group (mean change, each pain intensity at rest and during 
Table 2. Scores Recorded for the Subjects before and after the Intervention

\begin{tabular}{cccccccc}
\hline & & \multicolumn{3}{c}{ IFC Group } & \multicolumn{3}{c}{ Placebo Group } \\
\hline \multicolumn{2}{c}{ Variables } & Pre & Post & Change & Pre & Post & Change \\
\multirow{2}{*}{ VAS } & Resting & $5.40 \pm 0.88$ & $4.35 \pm 1.09$ & $-1.05 \pm 0.83^{*}$ & $4.92 \pm 1.00$ & $4.50 \pm 0.89$ & $-0.45 \pm 0.76^{* *}$ \\
& Trunk Flexion & $6.35 \pm 1.09$ & $5.55 \pm 1.23$ & $-0.80 \pm 1.70^{*}$ & $6.10 \pm 1.10$ & $5.90 \pm 1.20$ & $-0.20 \pm 0.77 * \#$ \\
\multicolumn{2}{c}{ Barthel Index } & $60.60 \pm 10.67$ & $63.65 \pm 9.72$ & $-2.85 \pm .89^{*}$ & $61.90 \pm 10.10$ & $63.95 \pm 10.23$ & $2.05 \pm 1.85^{*}$ \\
& ODI & $48.65 \pm 9.20$ & $45.80 \pm 8.37$ & $-2.85 \pm .89^{*}$ & $48.35 \pm 8.39$ & $47.05 \pm 8.29$ & $-1.30 \pm 2.27 * \#$ \\
& MCS & $34.85 \pm 13.82$ & $38.40 \pm 12.80$ & $3.55 \pm 4.65^{*}$ & $35.50 \pm 8.18$ & $36.20 \pm 7.40$ & $0.70 \pm 2.43 \#$ \\
\multirow{2}{*}{ Sf-36 } & PCS & $32.55 \pm 11.45$ & $36.25 \pm 10.99$ & $3.70 \pm 4.13^{*}$ & $34.10 \pm 7.72$ & $35.60 \pm 7.50$ & $1.50 \pm 1.50^{*} \#$ \\
\hline
\end{tabular}

VAS, visual analogue scale; SF36, short form 36 questionnaire; ODI, Oswestry disability index for low back pain; MCS, mental component score; PCS, physical component score

Values are expressed as the mean \pm standard deviation (SD)

movements than in the placebo stimulation group (Table 2). No significant differences in Barthel index were found between the IFC and placebo stimulation groups. The ODI score was significantly lower in the IFC group than the placebo stimulation group. The treatment caused a more significant increase in the MCS and PCS of SF 36 in the IFC group than in the placebo stimulation group (Table 2).

\section{Discussion}

This study examined the effects of IFC on pain in stroke patients with lumbago. The results revealed a significant decrease in pain in the IFC group (the post-test score was less than the pretest score: $12.7 \%$ and $20.4 \%$, respectively) compared to the control group (the posttest score was less than the pretest score: $3.2 \%$ and $8.1 \%$, respectively). In a study examining the analgesic effects of IFC in patients with chronic lumbago, the threshold for pressure pain increased significantly after a single application of IFC [27].

Moreover, the group that received IFC with concurrent lumbar region massage showed improvements in their resting pain, disability, and HRQoL [18]. Two hypotheses can explain the pain reduction of IFC: the release of endogenous opioids and the gate control theory of pain [28]. In large-diameter myelinated afferent nerves $(A \beta$ fibers), the action potential occurs at approximately 100 $\mathrm{Hz}$. Therefore, the A $\mathrm{B}$ fibers stimulated by IFC could block the type $\mathrm{C}$ nociceptive fibers (small-diameter non-myelinated afferent nerves), which deliver pain information, resulting in analgesia [16].

In stroke patients, pain can be initiated for various reasons, such as motor weakness, spasticity, restricted range of motion, altered neuromuscular control, proprioceptive deficits, disturbed and improper lower extremity kinematics, and gait biomechanics, and overuse of the connective tissues $[4,29,30]$. This type of pain slows motor recovery and impairs the activities of daily living [29,30,34-38]. Therefore, this study not only measured the resting pain but also compared the levels of pain during the functional movements for daily living. These findings suggest that the IFC application improved the pain intensity significantly while at rest and during movements (such as bending over while in standing).

Functional improvement is one of the main goals of stroke rehabilitation. Therefore, this study examined the effects of IFC on functional disability in the current study. The Barthel Index was used to measure the activities of daily living in stroke patients, and the ODI was used to assess the functional disabilities in lumbago patients. [22, 23]. The ODI improved significantly after the intervention 
(the post-test score was less than the pretest score IFC group: $2.1 \%$ and $5.8 \%$, respectively). The ODI is a measure of functional disability in relation to pain during rest and during the performance of various activities, such as sitting, standing, and walking [22]. Thus, the improvement in functional disability in the IFC group might be due to the reduction in pain during the functional movements after treatment. Suriya-amarit et al. [31] reported that IFC on shoulder pain in stroke patients reduced the pain intensity and improved the passive range of motion of the shoulder. Alternatively, the Barthel Index measures the dependency on activities of daily living (such as personal hygiene, food intake, and transfer) with regard to pain. Therefore, the intervention time in the present study might have been too short to observe any significant changes in this dependency.

A significant improvement in the HRQoL score was noted when the effects of IFC on the HRQoL were measured using the SF-36 in the current study (the posttest score was greater than the pretest score in MCS- $10.3 \%$, PCS-11.4\%, respectively, in the IFC group). Pain and negative emotions can be an obstacle to healthy living in stroke patients [32]. In addition, several studies have demonstrated significant associations between pain and muscle stiffness or spasm as well as depression. Coban et al. [33] reported that IFC could relieve the symptoms of pain and improve the patients' HRQoL. The decrease in lumbago and functional disability indicates that IFC improved the HRQoL of the patients in the current study.

This study reported that the application of IFC improved the pain, functional ability, and HRQoL in stroke patients with lumbago. On the other hand, only a limited number of participants were included in the study. Moreover, they were not followed up to determine the long-term effects of this intervention. Additional studies examining the effects of IFC in conjunction with concurrent massage or exercise in stroke patients with pain are warranted. Furthermore, the effects of IFC on functional improvement using various tools to assess the levels of spasticity and motor function are needed.

\section{Conclusion}

Based on the results of this study, an IFC treatment, which is easy to apply and has few side effects compared to drug intervention, might decrease the levels of pain and disability and improve the balance ability in stroke patients with chronic low back pain in clinics.

\section{Acknowledgements}

This study was supported by a National Research Foundation of Korea (NRF) grant funded by the Korea government (MSIT) (No. 2019027909).

\section{References}

[1] Harvey RL, Roth EJ, Yu D. Rehabilitation in stroke syndromes. In: Braddom RL, editor. Scand J Caring Sci. 3rd ed. Philadelphia, PA: Elsevier Inc. 2007;1175-212.

[2] Brandstater ME. Stroke rehabilitation. In: De Lisa JA, Gans BM, editors. Rehabilitation medicine principles and practice. Philadelphia, PA: Lippincott-Raven. 2005; 1656-76.

[3] Klit H, Finnerup NB, Overvad K, et al. Pain following stroke: a population-based follow-up study. Plos One. 2011;6:e27607.

[4] Hansen AP, Marcussen NS, Klit H, Andersen G, et al. Pain following stroke: A prospective study. Eur J Pain. 2012;16:1128-36.

[5] Jonsson A-C, Lindgren I, Hallström B, et al. Prevalence and intensity of pain after stroke: a population based study focusing on patients' perspectives. J Neurol Neurosurg Psychiatry. 2006; 77:590-5.

[6] Sackley CS, Brittle N, Patel S, et al. The prevalence of joint contractures, pressure sores, painful shoulder, 
other pain, falls and depression in the year after a severely disabling stroke. Stroke. 2008;39:3329-34.

[7] Andersen G, Vestergaard K, Ingeman-Nielsen M, et al. Incidence of central post-stroke pain. Pain. 1995;61: 187-93.

[8] Lin CH, Chen KH, Chang CH, et al. Muscle pain intensity and pressure pain threshold changes in different periods of stroke patients. Am J Phys Med Rehabil. 2014;93: 299-309.

[9] Van Bragt PJ, Van Ginneken BT, Westendorp T, et al. Predicting outcome in a postacute stroke rehabilitation programme. Int J Rehabil Res. 2014;37:110-7.

[10] Ekman M, Johnell O, Lidgren L. The economic cost of low back pain in Sweden in 2001. Acta Orthop . 2005;76:275-84.

[11] Köseoğlu BF, Akselim S, Kesikburun B, et al. The impact of lower extremity pain conditions on clinical variables and health-related quality of life in patients with stroke. Top Stroke Rehabil. 2017; 24:50-60.

[12] Facci LM, Nowotny JP, Tormem F, et al. Trevisani VF. Effects of transcutaneous electrical nerve stimulation (TENS) and interferential currents (IFC) in patients with nonspecific chronic low back pain: Randomized clinical trial. Sao Paulo Med J. 2011;129:206-16.

[13] Pantaleão MA, Laurino MF, Gallego NL, et al. Adjusting pulse amplitude during transcutaneous electrical nerve stimulation (TENS) application produces greater hypoalgesia. J Pain. 2011;12:581-90.

[14] Bae YH, Lee SM. Analgesic effects of transcutaneous electrical nerve stimulation and interferential current on experimental ischemic pain models: Frequencies of 50 Hz and 100Hz. J Phys Ther Sci. 2014;26:1945-8.

[15] Hurley DA, Minder PM, McDonough SM, et al. Interferential therapy electrode placement technique in acute low back pain: a preliminary investigation. Arch Phys Med Rehabil. 2001;82: 485-93.

[16] Hurley DA, McDonough SM, Dempster M, et al. A randomized clinical trial of manipulative therapy and interferential therapy for acute low back pain. Spine. 2004;29:2207-16.

[17] Suriya-amarit D1, Gaogasigam C1, Siriphorn A1, Boonyong S2. Effect of interferential current stimulation in management of hemiplegic shoulder pain. Arch Phys Med Rehabil. 2014;95:1441-6.

[18] Lara-Palomo IC, Aguilar-Ferrandiz ME, MataranPenarrocha G A, et al. Short-term effects of interferential current electro-massage in adults with chronic nonspecific low back pain: a randomized controlled trial. Clin Rehabil. 2013;27:439-49.

[19] Albornoz-Cabello M, Maya-Martín J, DomínguezMaldonado G, et al. Effect of interferential current therapy on pain perception and disability level in subjects with chronic low back pain: a randomized controlled trial. Clin Rehabil. 2017;31:242-9.

[20] Serrano-Atero MS, Caballero J, Cañas A, et al. Pain assessment (I). Rev Soc Esp Dolor. 2002;9: 94-108.

[21] Yarnitsky D, Sprecher E, Zaslansky R. Multiple session experimental pain measurements. Pain. 1996;67:327-33.

[22] Mahoney F, Barthel D. Functional evaluation: The Barthel Index. Md State Med J. 1965;14:61-5.

[23] Shah S, Vanclay F, Cooper B. Improving the sensitivity of the Barthel Index for stroke rehabilitation. J Clin Epidemiol. 1989;42:703-9.

[24] Alcantara-Bumbiedro S, Florez-Garcia MT, EchavarriPerez C, et al. Escala de incapacidad por dolor lumbar de Oswestry. Rehabilitación (Madr). 2006;40:150-8.

[25] Kim DY, Lee SH, Lee HY, et al. Validation of the Korean version of the Oswestry disability index. Spine (Phila Pa 1976). 2005;30:E123-7.

[26] Hisli N. Beck depresyon envanterinin geçerliği üzerine bir çalışma. Psikoloji Dergisi. A study on the validity of Beck Depression Inventory. Turkish Journal Psychology. 1988;6:118-26.

[27] Fuentes J, Armijo-Olivo S, Funabashi M, et al. Enhanced therapeutic alliance modulates pain intensity and muscle pain sensitivity in patients with chronic low back pain: 
an experimental controlled study. Phys Ther. 2014;94: 477-89

[28] Beatti A, Trucker K, Chipchase L. A double-blind placebo-controlled investigation into the effects of interferential therapy on experimentally induced pain using a cross-over design. Int Musculoskelet Med. 2012;34:115-22.

[29] Kong KH, Woon VC, Yang SY. Prevalence of chronic pain and its impact on health-related quality of life in stroke survivors. Arch Phys Med Rehabil. 2004;85:35-40.

[30] Kendall R. Musculoskeletal problems in stroke survivors. Top Stroke Rehabil. 2010;17:173-8.

[31] Suriya-amarit D, Gaogasigam C, Siriphorn A, Boonyong S. Effect of interferential current stimulation in management of hemiplegic shoulder pain. Arch Phys Med Rehabil. 2014 ;95:1441-6.

[32] Lennon OCI, Doody C, NiChoisdealbh C, et al. Barriers to healthy-lifestyle participation in stroke: consumer participation in secondary prevention design. Int $\mathrm{J}$ Rehabil Res. 2013;36:354-61.
[33] Coban S, Akbal E, Köklü S, et al. Clinical trial: transcutaneous interferential electrical stimulation in individuals with irritable bowel syndrome - a prospective doubleblind randomized study. Digestion. 2012;86:86-93.

[34] Dromerick AW, Edwards AF, Kumar A. Hemiplegic Shoulder Pain Syndrome: Frequency and Characteristics During Inpatient Stroke Rehabilitation. Arch Phys Med Rehabil. 2008;89:1589-93.

[35] Klit H, Finnerup NB, Jensen TS. Central post-stroke pain: clinical characteristics, pathophysiology, and management. Lancet Neurol. 2009;8:857-68.

[36] Koog Yun Hyung, Jin SS, Yoon K, Min B. Interventions for hemiplegic shoulder pain: systematic review of randomized controlled trials. Disabil Rehabil. 2010; 32:282-91

[37] Kumar G, Soni CR. Central post-stroke pain: current evidence. J Neurol Sci. 2009;284:10-17.

[38] Niessen MH, Veeger DH, Meskers CG, et al. Relationship among shoulder proprioception, kinematics and pain after stroke. Arch Phys Med Rehabil. 2009;90:1557-64. 